\title{
PARÂMETROS CINÉTICOS DA PIRÓLISE DE RESÍDUO DE COCO
}

\author{
CUNHA, L. B ${ }^{1}$, L. D. M. S. BOREL ${ }^{1}$, T.S. LIRA ${ }^{2}$, C.H. ATAÍDE ${ }^{1}$ e M. A. S. BARROZO ${ }^{1}$ \\ ${ }^{1}$ Universidade Federal de Uberlândia, Faculdade de Engenharia Química \\ ${ }^{2}$ Universidade Federal do Espírito Santo, Departamento de Engenharias e Tecnologia \\ E-mail para contato: leobragacunha@ hotmail.com
}

\begin{abstract}
RESUMO - Neste trabalho, foi avaliado o potencial do resíduo de coco como fonte de biomassa para produção de biocombustíveis por pirólise considerando suas propriedades físico-químicas e a cinética de degradação térmica. Foram realizadas análises termogravimétricas com temperaturas de 300 a $900 \mathrm{~K}$ e taxas de aquecimento entre 5 e $30 \mathrm{~K} / \mathrm{min}$, em condições inertes. Os parâmetros cinéticos do processo de decomposição foram estimados por métodos isoconversionais e pelo modelo de reações paralelas independentes (RPI). Os valores médios de energia de ativação $\left(E_{a}\right)$ calculados estão entre 133,3 e $146,3 \mathrm{~kJ} \mathrm{~mol}^{-1}$.
\end{abstract}

\section{INTRODUÇÃO}

São produzidos anualmente $62 \mathrm{Mt}$ de coco (Cocos nucifera L.), sendo o Brasil o quarto maior produtor (Siengchum et al., 2013). As aplicações para essa matéria-prima são diversas, incluindo indústrias alimentícias e de cosméticos, agricultura e artesanato. No entanto, grande parte torna-se resíduo. As cascas do coco correspondem a $70 \%$ do lixo produzido no litoral brasileiro devido à venda da água de coco. Já as indústrias rejeitam $80-85 \%$ do peso de fruto. Portanto, a destinação adequada deste resíduo constitui um problema ambiental devido ao tempo necessário para sua degradação e também ao favorecimento do desenvolvimento do mosquito Aedes aegypti (Corradini et al., 2009).

A pirólise é uma alternativa interessante para agregar valor a estes resíduos através da produção de gases, líquidos e sólidos combustíveis. As características físicas e químicas da biomassa afetam a qualidade e o rendimento dos produtos pirolíticos. Além disso, conhecer os parâmetros cinéticos da sua degradação térmica é essencial a fim de se obter melhores resultados. Diante deste contexto, o objetivo deste trabalho foi estudar a decomposição térmica do resíduo de coco empregando modelos cinéticos como o isoconversionais e o Modelo de Reações Paralelas Independentes (RPI) a fim de avaliar o uso deste material como fonte de biomassa para a produção de bio-óleo por pirólise.

\section{MATERIAIS E MÉTODOS}

\subsection{Materiais}

Os resíduos de coco utilizados foram obtidos no Parque do Sabiá em Uberlândia, MG $\left(18^{\circ} 55^{\prime} \mathrm{S}\right.$ e $\left.48^{\circ} 17^{\prime} \mathrm{O}\right)$. As amostras foram preparadas pela abertura do fruto e extração do 
endocarpo. Em seguida, o endocarpo foi moído, seco em estufa $\left(105 \pm 3^{\circ} \mathrm{C}\right)$ e separado via peneiramento $\left(0,15<d_{p}<0,3 \mathrm{~mm}\right)$. Procedeu-se então a determinação da composição elementar, utilizando um analisador Perkin Elmer $2400 \mathrm{CHNS} / \mathrm{O}$; da composição imediata, segundo as normas ASTM E1756-01, E872-82 e E1755-01 para umidade, voláteis e cinzas, respectivamente e da composição química em termos de extrativos, hemicelulose, celulose e lignina segundo a metodologia proposta por Morais et al. (2010).

\subsection{Análises termogravimétricas (TGA)}

As análises termogravimétricas foram realizadas no Laboratório de Análises Químicas do Programa de Pós-graduação em Energia, na Universidade Federal do Espírito Santo. Utilizou-se um analisador termogravimétrico (modelo DTG-60H, Shimadzu) e a análise foi realizada em atmosfera inerte de $\mathrm{N}_{2}(50 \mathrm{~mL} / \mathrm{min})$, de 303 a $1073 \mathrm{~K}$ com taxas de aquecimento de 5, 10, 20 e $30^{\circ} \mathrm{C} / \mathrm{min}$. A massa inicial das amostras foi de 5,4 $\pm 0,8 \mathrm{mg}$.

Dos dados obtidos, calculou-se a conversão conforme a Equação (1):

$$
X=\frac{m_{0}-m_{t}}{m_{0}-m_{\infty}}
$$

Sendo $m_{0}$ a massa inicial da amostra, $m_{\infty}$ a massa final e $m_{t}$ a massa em um dado tempo instantâneo.

Os parâmetros cinéticos da devolatização (423-1073K) foram determinados por métodos isoconversionais e modelos de reações paralelas independentes (RPI).

Os métodos isoconversionais envolvem a regressão linear dos dados e são capazes de fornecer resultados satisfatórios acerca da energia de ativação $\left(\mathrm{E}_{\mathrm{a}}\right)$ do processo (Santos et al, 2012). Os modelos utilizados neste trabalho estão apresentados na Tabela 1.

Tabela 1 - Equações dos métodos isoconversionais.

\begin{tabular}{cc}
\hline Modelo & Equação \\
\hline Friedman & $\ln [d X / d t]=\ln f(X)+\ln k_{0}-\left[E_{a} / R \rrbracket[1 / T]\right.$ \\
Kissinger & $\ln \left(\frac{\beta}{T^{2}}\right)=-\left(\frac{E_{a}}{R T}\right)+\left[\ln \left(\frac{k_{0} R}{T}\right)-\ln g(x)\right]$ \\
\hline
\end{tabular}

O modelo de reações paralelas independentes (RPI) considera a degradação individual de cada subcomponente. A taxa de conversão para cada componente pode ser escrita da seguinte forma:

$$
\frac{d X}{d t}=k_{o i} \exp \left(-\frac{E_{a i}}{R T}\right)\left(1-X_{i}\right)^{n_{i}}
$$


em que $X_{i}, k_{0 i}, E_{a i}$ e $n_{i}$ são, respectivamente, a conversão, o fator pré-exponencial $\left(\mathrm{s}^{-1}\right)$, a energia de ativação $(\mathrm{kJ} / \mathrm{mol})$ e a ordem aparente de reação de cada subcomponente.

Neste trabalho foram considerados quatro componentes: extrativos, celulose, hemicelulose e lignina. A taxa de reação total é obtida pela soma das taxas de reações parciais associadas às suas correspondentes frações mássicas. A perda de massa com relação ao tempo é calculada pela Equação (5):

$$
\frac{d m^{\text {calc }}}{d t}=-\left(m_{o}-m\right) \sum_{i=1}^{3} c_{i} \frac{d X_{i}^{n_{i}}}{d t}
$$

Em que $c_{i}$ é a fração em massa de cada subcomponente.

Os parâmetros cinéticos foram estimados utilizando o método de evolução diferencial implementado em MATHLAB R2013a (Lobato et al., 2008). Para isso, considerou-se a decomposição dos extrativos, hemicelulose e celulose como reações de $1^{\mathrm{a}}$ ordem, enquanto a lignina segue um mecanismo de $3^{\mathrm{a}}$ ordem (Alvarenga et al., 2016).

\section{RESULTADOS E DISCUSSÃO}

Na Tabela 2, é apresentada a composição química do resíduo de coco. Verificou-se que o resíduo de coco possui alto teor de voláteis e elevado poder calorífico.

Tabela 2 - Composição química do resíduo de coco, b.s..

\begin{tabular}{cccccc}
\hline $\begin{array}{c}\text { Composição elementar } \\
(\mathbf{\%} \mathbf{m} / \mathbf{m})\end{array}$ & \multicolumn{2}{c}{$\begin{array}{c}\text { Composição imediata } \\
(\% \mathbf{m} / \mathbf{m})\end{array}$} & \multicolumn{2}{c}{ Composição química $(\% \mathbf{~ m} / \mathbf{m})$} \\
\hline $\mathbf{C}$ & $46,33 \pm 0,17$ & Umidade & $6,88 \pm 0,04$ & Extrativos & $5,57 \pm 0,07$ \\
$\mathbf{H}$ & $5,93 \pm 0,02$ & Cinza & $2,78 \pm 0,02$ & Lignina & $32,44 \pm 3,68$ \\
$\mathbf{N}$ & $1,98 \pm 0,13$ & Voláteis & $81,65 \pm 0,31$ & Celulose & $31,06 \pm 0,94$ \\
$\mathbf{S}$ & $0,93 \pm 0,01$ & Carbono Fixo & $15,57 \pm 0,30$ & Hemicelulose & $30,93 \pm 0,94$ \\
& & & & 18,2 & \\
\hline
\end{tabular}




\section{Congresso Brasileiro de Engenharia Química em Iniciação Científica UFSCar - São Carlos - SP 16 a 19 de Julho de 2017}

Dos ensaios termogravimétricos foram obtidas curvas apresentadas na Figura 1. O perfil de decomposição obtido foi semelhante para as diferentes taxas de aquecimento.

Figura 1 - Curvas experimentais: (a) TG; e (b) DTG do endocarpo de coco.

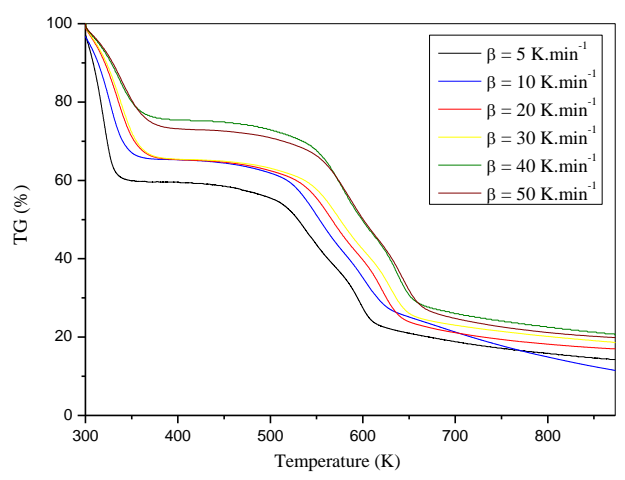

(a)

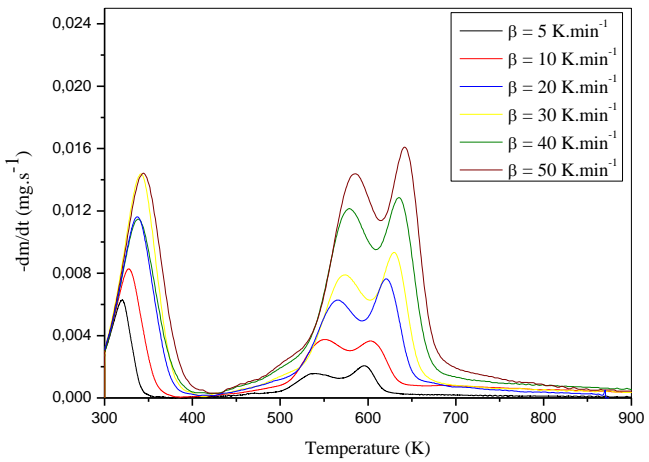

(b)

Na curva de TG (Figura 1(a)), tem-se a secagem da biomassa (até 400K) seguida da devolatilização (400-900K), etapa em que se registra a maior perda de massa. Nas curvas DTG (Figura 1(b)), o primeiro pico indica a perda de água. Os dois picos seguintes representam a degradação da hemicelulose $(525-580 \mathrm{~K})$ e da celulose $(590-660 \mathrm{~K})$. Acima de 660K, a degradação da lignina prossegue contínua e suavemente (SANTOS et al., 2012). Além disso, os extrativos presentes no material também influenciam sua taxa de degradação térmica global. O cálculo de $E_{a}$ por métodos isoconversionais (Tabela 1) foi realizado para $0,03<\mathrm{X}<0,90$. Na Figura 2, são apresentados os resultados das regressões lineares.

Figura 2: Resultados das regressões lineares usando (a) Friedman e (b) Kissinger.

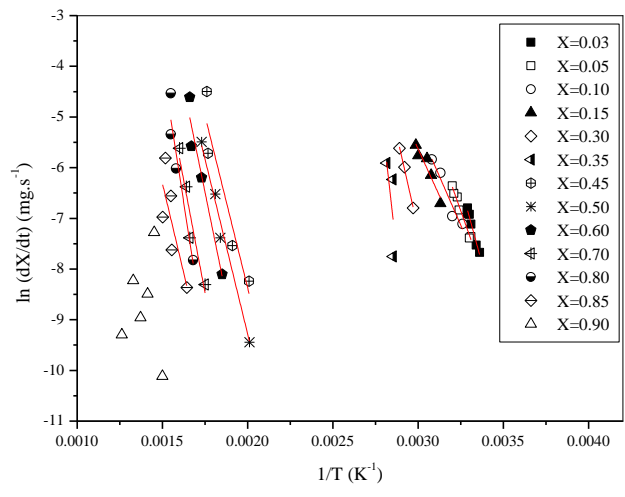

(a) Friedman

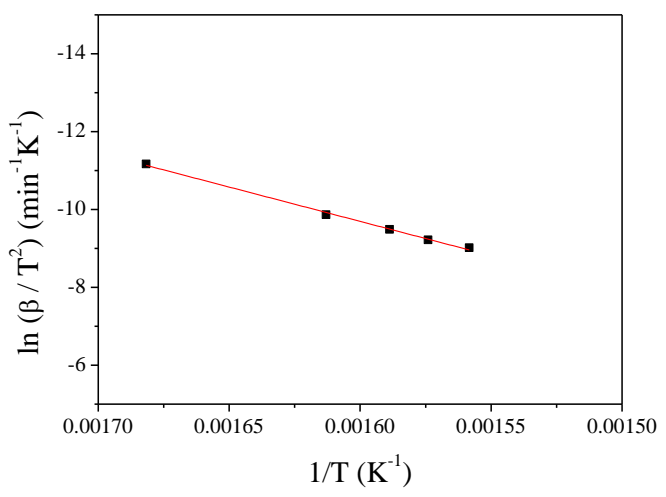

(b) Kissinger

$\mathrm{Na}$ abordagem isoconversional, as linhas paralelas da regressão indicam que as reações seguem o mesmo mecanismo para o intervalo de conversão. O não paralelismo da linha para conversões acima de 90\% (Figura 2(a)), indica que há mudanças no mecanismo e, consequentemente, diferentes valores de $E_{a}$. Tal fato pode ser atribuído à degradação da lignina, que segue uma cinética de terceira ordem. Utilizando o método de Friedman, obtevese uma $E_{a}$ média de $133,3 \mathrm{~kJ} / \mathrm{mol}\left(\mathrm{R}^{2}=0,9236\right)$, enquanto pelo método de Kissinger $E_{a}$ foi de 
$146,3 \mathrm{~kJ} / \mathrm{mol}\left(\mathrm{R}^{2}=0,9963\right)$. A Figura 3 mostra curvas DTG para uma taxa de aquecimento de $30 \mathrm{~K} \mathrm{~min}^{-1}$ :

Figura 3: Curvas DTG experimental e simulada pelo modelo RPI

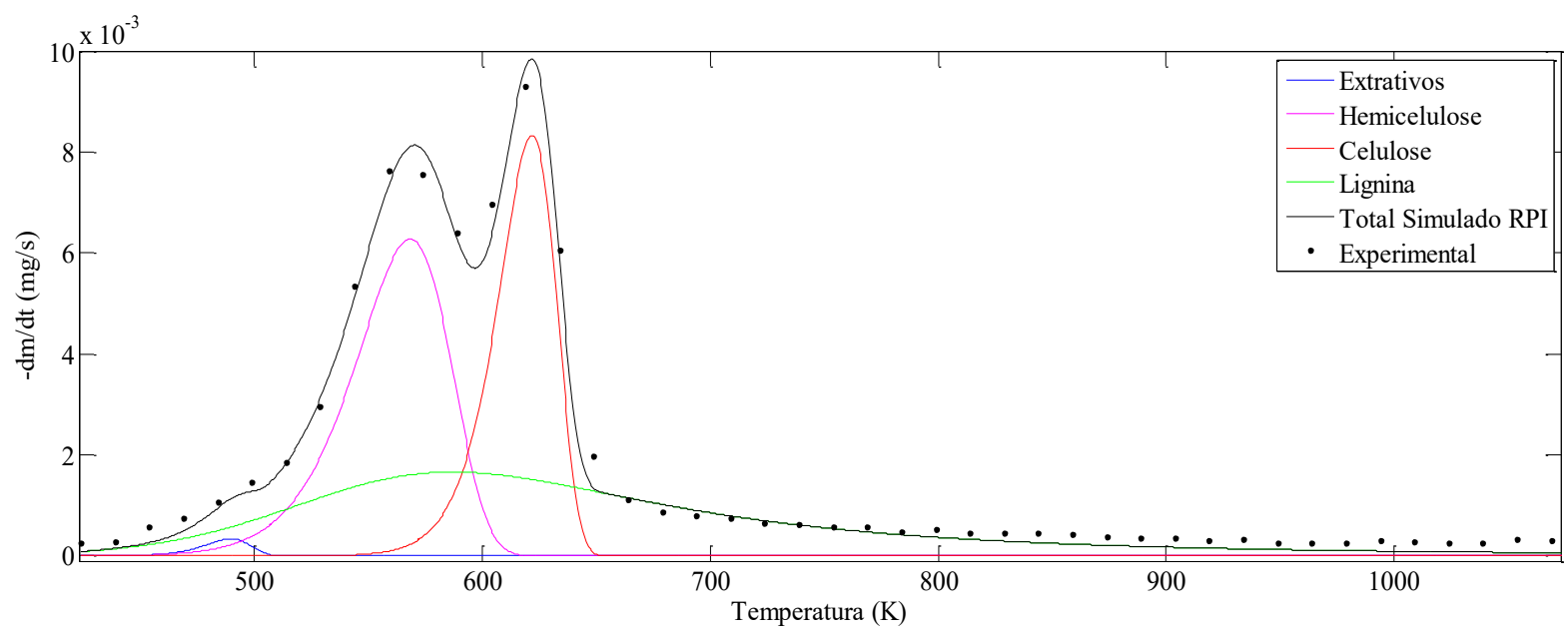

A Tabela 3 apresenta os parâmetros cinéticos e as frações de massa estimadas.

Tabela 3 - Resultados do modelo RPI para pirólise do endocarpo de coco.

\begin{tabular}{ccccccc}
\hline $\boldsymbol{\beta}(\mathbf{K} / \mathbf{m i n})$ & Parâmetros & Extrativos & Hemicelulose & Celulose & Lignina & $\begin{array}{c}\text { Desvio } \\
(\boldsymbol{\%})\end{array}$ \\
\hline \multirow{3}{*}{5} & $\boldsymbol{c}_{\boldsymbol{i}}$ & 0,07 & 0,28 & 0,26 & 0,32 & \\
& $\boldsymbol{k}_{\boldsymbol{0}}$ & $5,08 \cdot 10^{19}$ & $9,13 \cdot 10^{9}$ & $4,27 \cdot 10^{19}$ & $4,6 \cdot 10^{2}$ & 3,34 \\
& $\boldsymbol{E}_{\boldsymbol{a}}$ & 195,55 & 128,57 & 249,55 & 60,6 & \\
\multirow{2}{*}{10} & $\boldsymbol{c}_{\boldsymbol{i}}$ & 0,08 & 0,33 & 0,26 & 0,33 & \\
& $\boldsymbol{k}_{\boldsymbol{0}}$ & $5,26 \cdot 10^{19}$ & $3,18 \cdot 10^{9}$ & $1,49 \cdot 10^{19}$ & 8,67 & 5,87 \\
& $\boldsymbol{E}_{\boldsymbol{a}}$ & 197,65 & 122,81 & 244,06 & 63,95 & \\
& $\boldsymbol{c}_{\boldsymbol{i}}$ & 0,08 & 0,32 & 0,27 & 0,36 & \\
& $\boldsymbol{k}_{\boldsymbol{o}}$ & $3,36 \cdot 10^{19}$ & $7,39 \cdot 10^{9}$ & $3,46 \cdot 10^{19}$ & $5,62 \cdot 10^{2}$ & 3,65 \\
& $\boldsymbol{E}_{\boldsymbol{a}}$ & 194,67 & 126,21 & 250,5 & 56,59 & \\
& $\boldsymbol{c}_{\boldsymbol{i}}$ & 0,08 & 0,3 & 0,25 & 0,36 & \\
& $\boldsymbol{k}_{\boldsymbol{o}}$ & $3,74 \cdot 10^{19}$ & $8,21 \cdot 10^{9}$ & $3,84 \cdot 10^{19}$ & $4,18 \cdot 10^{2}$ & 2,68 \\
& $\boldsymbol{E}_{\boldsymbol{a}}$ & 195,85 & 125,66 & 249,97 & 53,57 & \\
\hline
\end{tabular}

A composição química da biomassa estimada usando o modelo RPI foi semelhante à determinação experimental (Tabela 1). Além disso, os valores de $E_{a}$ estimados estão de acordo com os dados da literatura (Alvarenga et al, 2016). O valor de $E_{a}$ médio obtido para a hemicelulose foi de $125,5 \pm 1,9 \mathrm{~kJ} \mathrm{~mol}^{-1}$, dentro da faixa de $80-150 \mathrm{kJmol}^{-1}$ relatada na literatura. Para a celulose, o valor médio obtido foi de $248,8 \pm 2,4 \mathrm{~kJ} \mathrm{~mol}^{-1}$, também dentro da 
faixa de $145-285 \mathrm{~kJ} \mathrm{~mol}^{-1}$. O valor médio estimado de $E_{a}$ para a lignina foi $56,8 \pm 4,6 \mathrm{~kJ} \mathrm{~mol}^{-}$

1. Para os extrativos, a $E_{a}$ média foi de $195,8 \pm 1,0 \mathrm{~kJ} \mathrm{~mol}^{-1}$.

\section{CONCLUSÃO}

Os resíduos de coco têm um bom potencial para a produção de bio-óleo devido ao alto teor de matéria volátil (81,65\% em peso) e HHV (18,2 MJ/kg). A cinética de desvolatilização foi estudada utilizando métodos isoconversionais e o modelo RPI. Valores semelhantes foram calculados para $E_{a}$ global por métodos de Friedman $(133,3 \mathrm{~kJ} / \mathrm{mol})$ e Kissinger $(146,3$ $\mathrm{kJ} / \mathrm{mol}$ ). Os valores de $E_{a}$ do modelo RPI para extrativos, hemicelulose, celulose e lignina foram 194,7-197,7, 122,8-128,6, 244,1-250,5 e 53,0-64,0 kJ/mol, respectivamente e são coerentes com os dados reportados na literatura para biomassa lignocelulósica. Além disso, as frações mássicas dos subcomponentes estimadas pelo modelo RPI foram próximas das medidas experimentais.

\section{REFERÊNCIAS}

ALVARENGA, L. M.; XAVIER, T. P.; BARROZO, M. A. S.; BACELOS, M. S.; LIRA, T. S. 2016. Determination of activation energy of pyrolysis of carton packaging wastes and its pure components using thermogravimetry. Waste Manage. 53, 68-75.

CORRADINI, E.; ROSA, M. F.; MACEDO, B. P.; PALADIN, P. D.; MATTOSO, L. H. C.. Composição química, propriedades mecânicas e térmicas da fibra de frutos de cultivares de coco verde. Revista Brasileira de Fruticultura. v.31. n.3. 2009. p. 837-846.

LOBATO Jr. F.S.; STEFFEN V.; ARRUDA, E.B.; BARROZO, M. A. S.; 2008. Estimation of drying parameters in rotary dryers using differential evolution. Journal of Physics Conference Series, 135, 1-8.

MORAIS, J. P. S.. Procedimentos para análise lignocelulósica./por João Paulo Saraiva Morais, Morsyleide de Freitas Rosa e José Manoel Marconcini.- Campina Grande: Embrapa Algodão, 2010. 54 p. (Embrapa Algodão/ Documentos, 236).

SANTOS, K.G., LIRA, T.S., GIANESELLA, M., LOBATO, F.S., MURATA, V.V., BARROZO, M.A.S. 2012. Bagasse pyrolysis: A comparative study of kinetic models. Chem. Eng. Commun.. 199 (1), p. 109-121.

SIENGCHUM, T.; ISENBERG, M.; CHUANG, S. S.C. Fast pyrolysis of coconut biomass An FTIR study. Fuel. v. 105. 2013. p. 559-565. 